Article

\title{
Certain Recurrence Relations of Two Parametric Mittag-Leffler Function and Their Application in Fractional Calculus
}

\author{
Dheerandra Shanker Sachan ${ }^{1}\left(\mathbb{D}\right.$, Shailesh Jaloree ${ }^{2}$ and Junesang Choi ${ }^{3, *(D)}$ \\ 1 Department of Mathematics, St.Mary's PG College, Vidisha 464001, India; sachan.dheerandra17@gmail.com \\ 2 Department of Mathematics, Samrat Ashok Technological Institute, Vidisha 464001, India; \\ shailesh_jaloree@rediffmail.com \\ 3 Department of Mathematics, Dongguk University, Gyeongju 38066, Korea \\ * Correspondence: junesangchoi@gmail.com; Tel.: +82-010-6525-2262
}

check for updates

Citation: Sachan, D.S.; Jaloree, S.; Choi, J. Certain Recurrence Relations of Two Parametric Mittag-Leffler Function and Their Application in Fractional Calculus. Fractal Fract. 2021, 5, 215. https://doi.org/ $10.3390 /$ fractalfract 5040215

Academic Editor: Maja Andrić

Received: 19 October 2021

Accepted: 10 November 2021

Published: 12 November 2021

Publisher's Note: MDPI stays neutral with regard to jurisdictional claims in published maps and institutional affiliations.

Copyright: (c) 2021 by the authors. Licensee MDPI, Basel, Switzerland. This article is an open access article distributed under the terms and conditions of the Creative Commons Attribution (CC BY) license (https:// creativecommons.org/licenses/by/ $4.0 /)$.

\begin{abstract}
The purpose of this paper is to develop some new recurrence relations for the two parametric Mittag-Leffler function. Then, we consider some applications of those recurrence relations. Firstly, we express many of the two parametric Mittag-Leffler functions in terms of elementary functions by combining suitable pairings of certain specific instances of those recurrence relations. Secondly, by applying Riemann-Liouville fractional integral and differential operators to one of those recurrence relations, we establish four new relations among the Fox-Wright functions, certain particular cases of which exhibit four relations among the generalized hypergeometric functions. Finally, we raise several relevant issues for further research.
\end{abstract}

Keywords: gamma function; Beta function; Mittag-Leffler function; Generalized Mittag-Leffler functions; generalized hypergeometric function; Fox-Wright function; recurrence relations; RiemannLiouville fractional calculus operators

MSC: 26A33; 32D15; 33B10; 33B15; 33C20; 33E12; 65Q30

\section{Introduction and Preliminaries}

Magnus Gösta Mittag-Leffler (1846-1927), a Swedish mathematician, invented the function $E_{\mu}(z)$ (1) in conjunction with the summation technique for divergent series, which is eponymously referred to as the Mittag-Leffler (M-L) function and represented by the following convergent power series across the whole complex plane (see [1-4]):

$$
E_{\mu}(z)=\sum_{n=0}^{\infty} \frac{z^{n}}{\Gamma(\mu n+1)} \quad(\Re(\mu)>0, z \in \mathbb{C}),
$$

where $\Gamma(\cdot)$ is the familiar Gamma function (see, e.g., ([5], [Section 1.1])). Let $\mathbb{C}, \mathbb{R}^{+} \mathbb{R}^{+}$, $\mathbb{Z}$, and $\mathbb{N}$ represent the sets of complex numbers, real numbers, positive real numbers, integers, and positive integers, respectively, in this and subsequent sections. Further, for some $\ell \in \mathbb{Z}$, let $\mathbb{Z}_{\leq \ell}$ denote the set of integers less than or equal to $\ell$. The function (1) is an entire function of order $1 / \Re(\mu)$ and type 1 (see, e.g., ([6], [Section 4.1])). Numerous mathematicians have investigated the properties of this entire function (see, e.g., [7-16]). This function (1) reduces to a number of elementary and special functions such as (see, e.g., ([6], [Section 3.2]))

$$
E_{1}( \pm z)=e^{ \pm z}, \quad E_{2}\left(-z^{2}\right)=\cos z, \quad E_{2}\left(z^{2}\right)=\cosh z,
$$

and

$$
E_{\frac{1}{2}}\left( \pm z^{\frac{1}{2}}\right)=e^{z}\left[1+\operatorname{erf}\left( \pm z^{\frac{1}{2}}\right)\right]=e^{z} \operatorname{erfc}\left(\mp z^{\frac{1}{2}}\right)
$$


where erf (erfc) represents the error function (complementary error function)

$$
\operatorname{erf}(z):=\frac{2}{\sqrt{\pi}} \int_{0}^{z} e^{-u^{2}} \mathrm{~d} u, \quad \operatorname{erfc}:=1-\operatorname{erf}(z) \quad(z \in \mathbb{C}),
$$

and $z^{\frac{1}{2}}$ denotes the principal branch of the associated multi-valued function. Numerous generalizations of the function (1) have been developed, including (5) and (7). In the 1960s, the Mittag-Leffler function began to be classified as a particular instance of the broader class of Fox $H$-function, which may have an arbitrary number of parameters in their Mellin-Barnes contour integral representation. Among the special instances of the $H$-function is the Fox-Wright function (8) (see, e.g., [10,17-19]). This function (1) and its numerous generalizations are significant because they are involved in a large number of applied problems (see, e.g., [20-27]). The Mittag-Leffler function (1) and its numerous extensions have been used, particulary, in conjunction with fractional calculus such as: The resolvant for a particular case of Volterra's equation (see ([28], [Theorem 4.2])) is explicitly expressed in terms of the Mittag-Leffler function (1); The two parametric Mittag-Leffler function $E_{\mu, \rho}(z)(5)$ and its slight extension are used as solutions of the linear integral equation of the Abel-Volterra type (see ([29], [Theorem 5.3])); Kilbas and Saigo ([30], [Theorems 6 and 7]) employed an extension of the Mittag-Leffler function as solutions of the Abel-Volterra integral equations (see also [31,32]). The monograph ([33], [pages $132,143,144,206])$ demonstrated in detail that the Mittag-Leffler function (1) and its modest modification are solutions to a certain class of fractional differential equations; the monograph ([34], [p. 21]) referred to the two parametric Mittag-Leffler function (5) and (1), as well as their fascinating Laplace transform formula. Choi et al. [35] proposed an extension of the Prabhakar function (7) that is used to determine a number of its features and formulae, including higher-order differential equations, many integral transformations, and several fractional derivative and integral formulas. Due to the breadth of applications in connection with fractional calculus, the Mittag-Leffler function has been dubbed the "Queen Function of the Fractional Calculus" by certain academics in the past (see [36]; see also [17]). Haubold et al. [24] provided a concise overview of the Mittag-Leffler function, extended Mittag-Leffler functions, Mittag-Leffler type functions, and their intriguing and useful features.

The two parametric Mittag-Leffler function $E_{\mu, \rho}(z)$ is defined by (see, e.g., [8,9], ([6], [Chapter 4]))

$$
E_{\mu, \varrho}(z)=\sum_{n=0}^{\infty} \frac{z^{n}}{\Gamma(\mu n+\varrho)} \quad(\Re(\mu)>0, \varrho, z \in \mathbb{C}) .
$$

The function (5) is an entire function of order $1 / \Re(\mu)$ and type 1 with the argument $z$ under the constraints $\mu, \varrho \in \mathbb{R}^{+}$(see, e.g., [37-39]), whose fundamental properties, such as asymptotic behavior and zero distribution, have been explored by a number of researchers (see, e.g., [8-11,40,41]). The function (5) reduces to some special functions such as (see, e.g., ([6], [Equation 4.3.13]))

$$
E_{1 / 2,1}(z)=e^{z^{2}}(1+\operatorname{erf} z)=e^{z^{2}} \operatorname{erfc}(-z) .
$$

The interested reader may refer to ([6], [Chapter 4]) for further basic properties and many other relations, representations and applications of the two parametric Mittag-Leffler function (5).

The three parametric Mittag-Leffler function (also known as the Prabhakar function [42]) $E_{\mu, Q}^{\tau}(z)$ is defined by (see [43]; see also ([6], [Chapter 5]), [42])

$$
E_{\mu, \varrho}^{\xi}(z)=\sum_{n=0}^{\infty} \frac{(\xi)_{n} z^{n}}{n ! \Gamma(\mu n+\varrho)} \quad(\Re(\mu)>0, \Re(\varrho)>0, \xi, z \in \mathbb{C}),
$$


which is also an entire function of order $1 / \Re(\mu)$ and type 1. Giusti et al. [42] offered an excellent survey paper in which they covered important findings and applications of the Prabhakar function (7), together with major historical events leading to its discovery and subsequent development, its capacity of introducing an upgraded scheme for fractional calculus, an overview of the advances made in applying this new general framework to physics and renewal processes, a collection of results on its numerical evaluation, and as many as 159 references.

The Mittag-Leffler function (1), its slight generalization (5), the Prabhakar function (7), and a number of other parameterized extensions are found to be particular instances of the following Fox-Wright function defined by (see [44-46]; see also [47], ([48], [p. 21]))

$$
{ }_{p} \Psi_{q}\left[\begin{array}{c}
\left(\alpha_{1}, A_{1}\right), \ldots,\left(\alpha_{p}, A_{p}\right) ; z \\
\left(\beta_{1}, B_{1}\right), \ldots,\left(\beta_{q}, B_{q}\right) ;
\end{array}\right]=\sum_{k=0}^{\infty} \frac{\prod_{j=1}^{p} \Gamma\left(\alpha_{j}+A_{j} k\right)}{\prod_{j=1}^{q} \Gamma\left(\beta_{j}+B_{j} k\right)} \frac{z^{k}}{k !},
$$

where the coefficients $A_{j} \in \mathbb{R}(j=1, \ldots, p)$ and $B_{j} \in \mathbb{R}(j=1, \ldots, q) ; \alpha_{j} \in \mathbb{C}(j=$ $1, \ldots, p)$ and $\beta_{j} \in \mathbb{C}(j=1, \ldots, q)$. The convergence constraints of (8) are given as follows (see ([49], [Theorem 1.5])): Let

$$
\begin{aligned}
& \Omega:=\sum_{j=1}^{q} B_{j}-\sum_{j=1}^{p} A_{j}, \\
& \omega:=\prod_{j=1}^{p}\left|A_{j}\right|^{-A_{j}} \prod_{j=1}^{q}\left|B_{j}\right|^{B_{j}}, \\
& v:=\sum_{j=1}^{q} \beta_{j}-\sum_{j=1}^{p} \alpha_{j}+\frac{p-q}{2} .
\end{aligned}
$$

Then

(i) If $\Omega>-1$, then the series (8) is absolutely convergent for all $z \in \mathbb{C}$;

(ii) If $\Omega=-1$, the series (8) is absolutely convergent for $|z|<\omega$;

(iii) If $\Omega=-1$ and $\Re(v)>\frac{1}{2}$, the series (8) is absolutely convergent for $|z|=\omega$.

The Fox-Wright function ${ }_{p} \Psi_{q}$ is an extension of generalized hypergeometric function ${ }_{p} F_{q}$ and a particular case of the $H$-function (see, e.g., ([19], [Equation (1.140)]), [50]):

$$
{ }_{p} \Psi_{q}\left[\begin{array}{l}
\left(\alpha_{1}, 1\right), \ldots,\left(\alpha_{p}, 1\right) ; \\
\left(\beta_{1}, 1\right), \ldots,\left(\beta_{q}, 1\right) ; z
\end{array}\right]=\frac{\prod_{j=1}^{p} \Gamma\left(\alpha_{j}\right)}{\prod_{j=1}^{q} \Gamma\left(\beta_{j}\right)} p F_{q}\left[\begin{array}{l}
\alpha_{1}, \ldots, \alpha_{p} ; \\
\beta_{1}, \ldots, \beta_{q} ;
\end{array}\right]
$$

and

$$
\begin{aligned}
{ }_{p} \Psi_{q} & {\left[\begin{array}{r}
\left(\alpha_{1}, A_{1}\right), \ldots,\left(\alpha_{p}, A_{p}\right) ; z \\
\left(\beta_{1}, B_{1}\right), \ldots,\left(\beta_{q}, B_{q}\right) ;
\end{array}\right] } \\
& =H_{p, q+1}^{1, p}\left[\begin{array}{r}
\left.-z \mid \begin{array}{r}
\left(1-\alpha_{1}, A_{1}\right), \ldots,\left(1-\alpha_{p}, A_{p}\right) \\
(0,1),\left(1-\beta_{1}, B_{1}\right), \ldots,\left(1-\beta_{q}, B_{q}\right)
\end{array}\right] .
\end{array}\right.
\end{aligned}
$$


There have been many introductions and investigations of fractional integrals and derivatives. We recall the left-sided and right-sided Riemann-Liouville fractional integrals $I_{a+}^{\alpha} f$ and $I_{b-}^{\alpha} f$ of order $\alpha \in \mathbb{C}$ defined as (see, e.g., $[33,34,49]$ )

$$
\left(I_{a+}^{\alpha} f\right)(x)=\frac{1}{\Gamma(\alpha)} \int_{a}^{x}(x-\tau)^{\alpha-1} f(\tau) d \tau \quad(x>a, \Re(\alpha)>0),
$$

and

$$
\left(I_{b-}^{\alpha} f\right)(x)=\frac{1}{\Gamma(\alpha)} \int_{x}^{b}(\tau-x)^{\alpha-1} f(\tau) d \tau \quad(b>x, \Re(\alpha)>0),
$$

respectively. The Riemann-Liouville fractional derivatives $D_{a+}^{\alpha} f$ and $D_{b-}^{\alpha} f$ of order $\alpha \in \mathbb{C}$ $(\Re(\alpha) \geq 0)$ are defined by

$$
\left(D_{a+}^{\alpha} f\right)(x)=\left(\frac{\mathrm{d}}{\mathrm{d} x}\right)^{\mathrm{n}}\left(I_{a+}^{\mathrm{n}-\alpha} f\right)(x) \quad(x>a)
$$

and

$$
\left(D_{b-}^{\alpha} f\right)(x)=\left(-\frac{\mathrm{d}}{\mathrm{d} x}\right)^{\mathrm{n}}\left(I_{b-}^{\mathrm{n}-\alpha} f\right)(x) \quad(x<b),
$$

where $\mathrm{n}=[\Re(\alpha)]+1$. Here and elsewhere, $[x]$ denotes the largest integer less than or equal to $x \in \mathbb{R}$.

We recall some of the recurrence relations for the two parametric Mittag-Leffler function (5) and the three parametric Mittag-Leffler function (7).

\subsection{Two Parametric Mittag-Leffler Function:}

$$
E_{\mu, \varrho}(z)=z E_{\mu, \mu+\varrho}(z)+\frac{1}{\Gamma(\varrho)}
$$

(see, e.g., ([10], [Equation (23)]), ([51], [Equation (5)])).

Further, for all $\mu, \varrho \in \mathbb{R}^{+}$,

$$
\begin{gathered}
E_{\mu, \varrho}(z)=z^{2} E_{\mu, \varrho+2 \mu}(z)+\frac{1}{\Gamma(\varrho)}+\frac{z}{\Gamma(\varrho+\mu)}, \\
E_{\mu, \varrho}(z)=z^{3} E_{\mu, \varrho+3 \mu}(z)+\frac{1}{\Gamma(\varrho)}+\frac{z}{\Gamma(\varrho+\mu)}+\frac{z^{2}}{\Gamma(\varrho+2 \mu)}, \\
E_{\mu, \varrho}(z)=z^{4} E_{\mu, \varrho+4 \mu}(z)+\frac{1}{\Gamma(\varrho)}+\frac{z}{\Gamma(\varrho+\mu)}+\frac{z^{2}}{\Gamma(\varrho+2 \mu)}+\frac{z^{3}}{\Gamma(\varrho+3 \mu)}
\end{gathered}
$$

(see, e.g., ([6], [Lemma 4.1]), [51]).

Generally, for $\Re(\mu)>0, \Re(\varrho)>0$, and $\ell \in \mathbb{N}$,

$$
E_{\mu, \varrho}(z)=z^{\ell} E_{\mu, \varrho+\ell \mu}(z)+\sum_{k=0}^{\ell-1} \frac{z^{k}}{\Gamma(\varrho+k \mu)}
$$

(see [52]; see also ([24], [Theorem 5.2])).

Gupta and Debnath (see ([51], [Equation (30)])) presented the following interesting differential recurrence relation for the two parametric Mittag-Leffler function (5):

$$
\begin{aligned}
E_{\mu, n+1}(z)= & n(n+2) E_{\mu, n+3}(z)+\mu z[\mu+2(n+1)] E_{\mu, n+3}^{\prime}(z) \\
& +z^{2} E_{\mu, n+3}^{\prime \prime}(z)+E_{\mu, n+2}(z) \quad(n \in \mathbb{N}),
\end{aligned}
$$


where

$$
E_{\mu, \varrho}^{(\ell)}(z)=\frac{\mathrm{d}^{\ell}}{\mathrm{d} z^{\ell}} E_{\mu, \varrho}(z) \quad\left(\ell \in \mathbb{N}_{0}\right) .
$$

The following recurrence relation reveals that, under the restrictions, computation of $E_{\mu, \rho}(z)$ for the case $\mu>1$ may be reduced to the case $0<\mu \leq 1$ (see, e.g., ([53], [Equation (6)]), ([10], [Chapter XVIII]), ([54], [p. 24]), ([55], [Equation (2.2)]), [56]):

$$
\begin{gathered}
E_{\mu, \varrho}(z)=\frac{1}{2 m+1} \sum_{k=-m}^{m} E_{\mu /(2 m+1), \varrho}\left(z^{1 /(2 m+1)} e^{2 \pi i k /(2 m+1)}\right) \\
\left(\mu \in \mathbb{R}^{+}, \varrho \in \mathbb{R}, z \in \mathbb{C}, m=[(\mu-1) / 2]+1\right) .
\end{gathered}
$$

1.2. Three Parametric Mittag-Leffler Function and Its Various Extensions

Giusti et al. pointed out the following interesting recurrence relations for the Prabhakar function (7) (see ([42], [Equations (4.2) and (4.3)])):

$$
E_{\mu, \varrho}^{\xi+1}(z)=\frac{E_{\mu, Q-1}^{\xi}(z)+(1-\varrho+\mu \xi) E_{\mu, \varrho}^{\xi}(z)}{\mu \xi}
$$

(see [43]) and

$$
E_{\mu, \varrho}^{\xi+1}(z)=\frac{E_{\mu, \varrho-\mu-1}^{\xi}(z)+(1-\varrho+\mu) E_{\mu, \varrho-\mu}^{\xi}(z)}{\mu \xi z}(z \in \mathbb{C} \backslash\{0\})
$$

(see [57]).

Shukla and Prajapati ([58], [Theorem 1]) offered an intriguing differential recurrence relation for an extended Mittag-Leffler function, which can be made by replacing $(\xi)_{n}$ in (7) with $(\xi)_{q n}$ under the restriction $q \in(0,1) \cup \mathbb{N}$ (see [59]), which was further generalized and investigated by Srivastava and Tomovski [50] who substituted a $k \in \mathbb{C}$ for the $q \in(0,1) \cup \mathbb{N}$ under constraints $\Re(\mu)>\max \{0, \Re(k)-1\}$ and $\Re(k)>0$.

Salim ([60], [Theorem 2.2]) presented two interesting differential recurrence relation for an extended Mittag-Leffler function, which can be derived by replacing $n$ ! in (7) with a Pochhammer symbol, say $(\eta)_{n}$ under the constraint $\Re(\eta)>0$.

Kurulay and Bayram ([61], [Theorems 4]) established an intriguing differential recurrence relation for the Prabhakar function (7).

Dhakar and Sharma ([62], [Theorem 2.1]) provided an interesting differential recurrence relation for the $k$-Mittag-Leffler function (see [63]), which may be obtained by substituting $(\xi)_{n, k}$ and $\Gamma_{k}(\mu n+\varrho)$ for $(\xi)_{n}$ and $\Gamma(\mu n+\varrho)$ in (7), respectively. Here the $k$-Pochhammer symbol is defined as follows (see [64]):

$$
(\gamma)_{n, k}:= \begin{cases}\frac{\Gamma_{k}(\gamma+n k)}{\Gamma_{k}(\gamma)} & \left(n \in \mathbb{N} ; k \in \mathbb{R}^{+} ; \gamma \in \mathbb{C} \backslash\{0\}\right), \\ \gamma(\gamma+k) \cdots(\gamma+(n-1) k) & (n \in \mathbb{N} ; \gamma \in \mathbb{C}),\end{cases}
$$

where $\Gamma_{k}$ is the $k$-gamma function defined by

$$
\Gamma_{k}(z)=\int_{0}^{\infty} e^{-\frac{t^{k}}{k}} t^{z-1} d t \quad\left(\Re(z)>0 ; k \in \mathbb{R}^{+}\right) .
$$

Sharma and Jain ([65], [Theorem 1]) gave an interesting recurrence relation for the $q$-analog (or extension) of the Prabhakar function (7). 
Gehlot ([66], [Theorem 2.1]) provided an intriguing differential recurrence relation for the following $p-k$ Mittag-Leffler function (see [67]):

$$
\begin{gathered}
{ }_{p} E_{k, \mu, \varrho}^{\xi, q}(z)=\sum_{n=0}^{\infty} \frac{p(\xi)_{n q, k} z^{n}}{n !_{p} \Gamma_{k}(\mu n+\varrho)} \\
\left(k, p \in \mathbb{R}^{+}, q \in(0,1) \cup \mathbb{N}, \min \{\Re(\mu), \Re(\varrho), \Re(\xi)\}>0\right) .
\end{gathered}
$$

Here the $p$ - $k$ Pochhammer symbol $p(\alpha)_{n, k}$ and the $p-k$ Gamma function ${ }_{p} \Gamma_{k}$ are defined by

$$
\begin{gathered}
p(\alpha)_{n, k}=\left(\frac{\alpha p}{k}\right)\left(\frac{\alpha p}{k}+p\right) \cdots\left(\frac{\alpha p}{k}+(n-1) p\right) \\
\left(k, p \in \mathbb{R}^{+}, n \in \mathbb{N}, \Re(\alpha)>0\right)
\end{gathered}
$$

and $p_{p}(\alpha)_{0, k}:=1$;

$$
{ }_{p} \Gamma_{k}(z)=\int_{0}^{\infty} e^{-\frac{t^{k}}{p}} t^{z-1} \mathrm{~d} t \quad\left(\Re(z)>0 ; p, k \in \mathbb{R}^{+}\right) .
$$

Further Gehlot ([67], [Theorem 2.3]) presented an interesting recurrence relation for the $p-k$ Mittag-Leffler function.

Choi et al. ([35], [Theorem 3.1]) established a differential recurrence relation for the extended Mittag-Leffler function, which may be obtained by replacing $(\xi)_{n}$ in $(7)$ with the generalized Pochhammer symbol $(\xi ; p)_{n}$. Here the generalized Pochhammer symbol $(\xi ; p)_{v}(\xi, v \in \mathbb{C})$ is defined by

$$
(\xi ; p)_{v}:= \begin{cases}\frac{\Gamma_{p}(\tilde{\xi}+v)}{\Gamma(\xi)} & (\Re(p)>0), \\ (\xi)_{v} & (p=0),\end{cases}
$$

where $\Gamma_{p}(z)$ is the generalized gamma function given as follows:

$$
\Gamma_{p}(z):= \begin{cases}\int_{0}^{\infty} t^{z-1} e^{-t-\frac{p}{t}} \mathrm{~d} t & (\Re(p)>0, z \in \mathbb{C}), \\ \Gamma(z) & (p=0, \Re(z)>0) .\end{cases}
$$

The aim of this article is to explore some new recurrence relations for the two parametric Mittag-Leffler function. Then, we discuss several applications of such recurrence relations. To begin, we express a number of the two parametric Mittag-Leffler functions in terms of elementary functions by combining appropriate pairings of particular instances of those recurrence relations. Second, we establish four new relations among the Fox-Wright functions by applying Riemann-Liouville fractional integral and differential operators to one of those recurrence relations. Certain particular cases of the Fox-Wright function relations exhibit four relations among the generalized hypergeometric functions. Finally, we propose several pertinent research questions.

Further, for our purpose, we recall the classical Beta function (see, e.g., ([5], [p. 8]))

$$
B(\alpha, \beta)= \begin{cases}\int_{0}^{1} \tau^{\alpha-1}(1-\tau)^{\beta-1} \mathrm{~d} \tau & (\Re(\alpha)>0, \Re(\beta)>0) \\ \frac{\Gamma(\alpha) \Gamma(\beta)}{\Gamma(\alpha+\beta)} & \left(\alpha, \beta \in \mathbb{C} \backslash \mathbb{Z}_{\leq 0}\right) .\end{cases}
$$

The following formula is one of a number of definite integrals that may be expressed in terms of the Beta function (see, e.g., ([5], [p. 9, Equation (49)])):

$$
\int_{a}^{b}(\tau-a)^{\alpha-1}(b-\tau)^{\beta-1} \mathrm{~d} \tau=(b-a)^{\alpha+\beta-1} B(\alpha, \beta)
$$




$$
(b>a, \Re(\alpha)>0, \Re(\beta)>0) .
$$

\section{Recurrence Relations}

This section explores some new recurrence relations for the two parametric MittagLeffler function (5).

Theorem 1. Let $\mu, \varrho, z \in \mathbb{C}$ with $\Re(\mu)>0$. Then

$$
\begin{aligned}
& E_{\mu, \varrho}(z)=\varrho(\varrho+1) E_{\mu, \varrho+2}(z)-\varrho(\varrho+1) z E_{\mu, \mu+\varrho+2}(z)+z E_{\mu, \mu+\varrho}(z) \quad(\Re(\varrho)>0) ; \\
& E_{\mu, \varrho}(z)=z^{3} E_{\mu, 3 \mu+\varrho}(z)-z^{2}(\mu+\varrho) E_{\mu, 2 \mu+\varrho+1}(z)+z(\mu+\varrho) E_{\mu, \mu+\varrho+1}(z) \\
& +\frac{z^{2}}{\Gamma(2 \mu+\varrho)}+\frac{1}{\Gamma(\varrho)} \quad(\Re(\varrho)>0) \text {; } \\
& (\varrho-1) E_{\mu, \varrho}(z)=z^{3} E_{\mu, 3 \mu+\varrho-1}(z)-z E_{\mu, \mu+\varrho-1}(z)+z(\varrho-1) E_{\mu, \mu+\varrho}(z) \\
& +\frac{z^{2}}{\Gamma(2 \mu+\varrho-1)}+\frac{z}{\Gamma(\mu+\varrho-1)}+\frac{1}{\Gamma(\varrho-1)} \quad(\Re(\varrho)>1) ; \\
& (\varrho-1) E_{\mu, \varrho}(z)=z(\varrho-1) E_{\mu, \mu+\varrho}(z)-z^{2} E_{\mu, 2 \mu+\varrho-1}(z)+\frac{1}{z} E_{\mu, \varrho-\mu-1}(z) \\
& -\frac{z}{\Gamma(\mu+\varrho-1)}-\frac{1}{z \Gamma(\varrho-\mu-1)} \quad(\Re(\varrho)>1, \Re(\varrho-\mu)>1) ; \\
& (\varrho-1) E_{\mu, \varrho}(z)=(\varrho-1) z^{2} E_{\mu, 2 \mu+\varrho}(z)-z^{2} E_{\mu, 2 \mu+\varrho-1}(z)+\frac{1}{z} E_{\mu, \varrho-\mu-1}(z) \\
& -\frac{1}{z \Gamma(\varrho-\mu-1)}-\frac{\mu z}{\Gamma(\mu+\varrho)} \quad(\Re(\varrho)>0, \Re(\varrho-\mu)>1) \text {; } \\
& (\varrho-2)(\varrho-1) E_{\mu, \varrho}(z)=z(\varrho-1)(\varrho-2) E_{\mu, \mu+\varrho}(z)+z^{3} E_{\mu, 3 \mu+\varrho-2}(z) \\
& -z E_{\mu, \mu+\varrho-2}(z)+\frac{z^{2}}{\Gamma(2 \mu+\varrho-2)}+\frac{z}{\Gamma(\mu+\varrho-2)}+\frac{1}{\Gamma(\varrho-2)} \quad(\Re(\varrho)>2) \text {; } \\
& (\mu+\varrho-2)(\mu+\varrho-1) E_{\mu, \varrho}(z)=(\mu+\varrho-2)(\mu+\varrho-1) z^{2} E_{\mu, 2 \mu+\varrho}(z) \\
& +z^{3} E_{\mu, 3 \mu+\varrho-2}(z)-z^{2} E_{\mu, 2 \mu+\varrho-2}(z) \\
& +\frac{z^{2}}{\Gamma(2 \mu+\varrho-2)}+\frac{z}{\Gamma(\mu+\varrho-2)}+\frac{(\mu+\varrho-2)(\mu+\varrho-1)}{\Gamma(\varrho)} \\
& (\Re(\varrho)>0, \Re(\varrho+\mu)>2) \text {. }
\end{aligned}
$$

Proof. We establish only (35). Let $\mathcal{R}_{1}$ be the right-handed member of (35). By using (5), we obtain

$$
\begin{aligned}
\mathcal{R}_{1}= & \varrho(\varrho+1) \sum_{k=0}^{\infty} \frac{z^{k}}{\Gamma(\mu k+\varrho+2)}-\varrho(\varrho+1) \sum_{k=0}^{\infty} \frac{z^{k+1}}{\Gamma(\mu k+\mu+\varrho+2)} \\
& +\sum_{k=0}^{\infty} \frac{z^{k+1}}{\Gamma(\mu k+\mu+\varrho)} .
\end{aligned}
$$


Setting $k+1=k^{\prime}$ on the second and third summations in (42) and dropping the prime on $k$, we obtain

$$
\begin{aligned}
\mathcal{R}_{1}= & \varrho(\varrho+1) \sum_{k=0}^{\infty} \frac{z^{k}}{\Gamma(\mu k+\varrho+2)}-\varrho(\varrho+1) \sum_{k=1}^{\infty} \frac{z^{k}}{\Gamma(\mu k+\varrho+2)}+\sum_{k=1}^{\infty} \frac{z^{k}}{\Gamma(\mu k+\varrho)} \\
= & \frac{\varrho(\varrho+1)}{\Gamma(\varrho+2)}+\sum_{k=1}^{\infty} \frac{z^{k}}{\Gamma(\mu k+\varrho)}=\frac{1}{\Gamma(\varrho)}+\sum_{k=1}^{\infty} \frac{z^{k}}{\Gamma(\mu k+\varrho)} \\
& =\sum_{k=0}^{\infty} \frac{z^{k}}{\Gamma(\mu k+\varrho)}=E_{\mu, \varrho}(z) .
\end{aligned}
$$

For the third equality of (43), the following fundamental relation for the Gamma function

$$
\Gamma(z+1)=z \Gamma(z)
$$

is used. The proof of (35) is complete.

Likewise, the remaining relations (36)-(41) may be established. We omit specifics.

Taking $\varrho=1$ in (35), (36), and (41), we obtain some relations between the MittagLeffler function (1) and the two parametric Mittag-Leffler function (5) in the following corollary.

Corollary 1. Let $\mu, z \in \mathbb{C}$ with $\Re(\mu)>0$. Then

$$
\begin{aligned}
E_{\mu}(z)= & 2 E_{\mu, 3}(z)-2 z E_{\mu, \mu+3}(z)+z E_{\mu, \mu+1}(z) ; \\
E_{\mu}(z)= & z^{3} E_{\mu, 3 \mu+1}(z)-z^{2}(\mu+1) E_{\mu, 2 \mu+2}(z) \\
& +z(\mu+1) E_{\mu, \mu+2}(z)+\frac{z^{2}}{\Gamma(2 \mu+1)}+1 ; \\
\mu(\mu-1) E_{\mu}(z)= & \mu(\mu-1) z^{2} E_{\mu, 2 \mu+1}(z)+z^{3} E_{\mu, 3 \mu-1}(z) \\
& -z^{2} E_{\mu, 2 \mu-1}(z)+\frac{z^{2}}{\Gamma(2 \mu-1)}+\frac{z}{\Gamma(\mu-1)}+\mu(\mu-1) .
\end{aligned}
$$

Similar to (2), the following corollary provides certain interesting expressions of several elementary functions in terms of the two parametric functions (5).

Corollary 2. Let $z \in \mathbb{C}$. Then

$$
\begin{gathered}
e^{z}=E_{1}(z)=z E_{1,2}(z)+2 E_{1,3}(z)-2 z E_{1,4}(z) \\
e^{z}=E_{1}(z)=2 z E_{1,3}(z)+\left(z^{3}-2 z^{2}\right) E_{1,4}(z)+\frac{z^{2}}{2}+1 \\
\cos z=E_{2}\left(-z^{2}\right)=\left(2-z^{2}\right) E_{2,3}\left(-z^{2}\right)+2 z^{2} E_{2,5}\left(-z^{2}\right) \\
\cos z=E_{2}\left(-z^{2}\right)=-3 z^{2} E_{2,4}\left(-z^{2}\right)-3 z^{4} E_{2,6}\left(-z^{2}\right) \\
-z^{6} E_{2,7}\left(-z^{2}\right)+\frac{z^{4}}{24}+1 ; \\
\cos z=E_{2}\left(-z^{2}\right)=-\frac{z^{4}}{2} E_{2,3}\left(-z^{2}\right)+\left(z^{4}-\frac{z^{6}}{2}\right) E_{2,5}\left(-z^{2}\right) \\
+\frac{z^{4}}{4}-\frac{z^{2}}{2}+1
\end{gathered}
$$




$$
\begin{aligned}
\sin z=z E_{2,2}\left(-z^{2}\right)= & \left(6 z-z^{3}\right) E_{2,4}\left(-z^{2}\right)+6 z^{3} E_{2,6}\left(-z^{2}\right) ; \\
\sin z=z E_{2,2}\left(-z^{2}\right)= & -4 z^{3} E_{2,5}\left(-z^{2}\right)-4 z^{5} E_{2,7}\left(-z^{2}\right) \\
& -z^{7} E_{2,8}\left(-z^{2}\right)+\frac{z^{5}}{120}+z ; \\
\sin z=z E_{2,2}\left(-z^{2}\right)= & z^{3} E_{2,3}\left(-z^{2}\right)-z^{3} E_{2,4}\left(-z^{2}\right) \\
& -z^{7} E_{2,7}\left(-z^{2}\right)+\frac{z^{5}}{24}-\frac{z^{3}}{2}+z ; \\
\sin z=z E_{2,2}\left(-z^{2}\right)=- & \frac{z^{5}}{6} E_{2,4}\left(-z^{2}\right)+\left(z^{5}-\frac{z^{7}}{6}\right) E_{2,6}\left(-z^{2}\right) \\
+ & \frac{z^{5}}{36}-\frac{z^{3}}{6}+z ; \\
\cosh z=E_{2}\left(z^{2}\right)= & \left(2+z^{2}\right) E_{2,3}\left(z^{2}\right)-2 z^{2} E_{2,5}\left(z^{2}\right) ; \\
\cosh z=E_{2}\left(z^{2}\right)= & 3 z^{2} E_{2,4}\left(z^{2}\right)-3 z^{4} E_{2,6}\left(z^{2}\right) \\
& +z^{6} E_{2,7}\left(z^{2}\right)+\frac{z^{4}}{24}+1 ; \\
+ & \frac{z^{5}}{36}+\frac{z^{3}}{6}+z . \\
\cosh z=E_{2}\left(z^{2}\right)=- & \frac{z^{4}}{2} E_{2,3}\left(z^{2}\right)+\left(z^{4}+\frac{z^{6}}{2}\right) E_{2,5}\left(z^{2}\right) \\
+ & \frac{z^{4}}{4}+\frac{z^{2}}{2}+1 ; \\
\sinh z=z E_{2,2}\left(z^{2}\right)=- & \frac{z^{5}}{6} E_{2,4}\left(z^{2}\right)+\left(z^{5}+\frac{z^{7}}{6}\right) E_{2,6}\left(z^{2}\right) \\
\sinh z=z E_{2,2}\left(z^{2}\right)= & -z^{3} E_{2,3}\left(z^{2}\right)+z^{3} E_{2,4}\left(z^{2}\right)+\frac{z^{3}}{24}+z \\
\sinh z=z E_{2,2}\left(z^{2}\right)= & \left.z^{3}\right) E_{2,4}\left(z^{2}\right)-6 z^{3} E_{2,6}\left(z^{2}\right) ; \\
\sinh z=z E_{2,2}\left(z^{2}\right)= & 4 z^{3} E_{2,5}\left(z^{2}\right)-4 z^{5} E_{2,7}\left(z^{2}\right) \\
& \\
&
\end{aligned}
$$

Proof. Setting $\mu=1$ in (45) and (46), respectively, yields (48) and (49).

Putting $\mu=2$ and replacing $z$ by $-z^{2}$ in (45), (46), and (47), respectively, gives (50), (51), and (52).

Taking $\mu=2, \varrho=2$ and replacing $z$ by $-z^{2}$ in (35), (36), (37), and (41), respectively, produces (53), (54), (55), and (56). and (59).

Setting $\mu=2$ and replacing $z$ by $z^{2}$ in (45), (46), and (47), respectively, offers (57), (58),

Putting $\mu=2, \varrho=2$ and replacing $z$ by $z^{2}$ in (35), (36), (37), and (41), respectively, affords (60), (61), (62), and (63).

By combining appropriate pairings of the identities in Corollary 2, such as (2), we may express several of the two parametric Mittag-Leffler functions in terms of elementary functions, as stated in the following corollary. 
Corollary 3. The following formulas hold.

$$
\begin{gathered}
E_{2,3}\left(-z^{2}\right)=-\frac{1}{z^{2}}(\cos z-1) \quad(z \in \mathbb{C} \backslash\{0\}) ; \\
E_{2,5}\left(-z^{2}\right)=\frac{1}{z^{4}}\left(\cos z+\frac{z^{2}}{2}-1\right) \quad(z \in \mathbb{C} \backslash\{0\}) ; \\
E_{2,4}\left(-z^{2}\right)=\frac{1}{z^{2}}\left(1-\frac{\sin z}{z}\right) \quad(z \in \mathbb{C} \backslash\{0\}) ; \\
E_{2,6}\left(-z^{2}\right)=\frac{1}{z^{4}}\left(-1+\frac{z^{2}}{6}+\frac{\sin z}{z}\right) \quad(z \in \mathbb{C} \backslash\{0\}) ; \\
E_{2,3}\left(z^{2}\right)=\frac{1}{z^{2}}(\cosh z-1) \quad(z \in \mathbb{C} \backslash\{0\}) ; \\
E_{2,5}\left(z^{2}\right)=\frac{1}{z^{4}}(\cosh z-1)-\frac{1}{2 z^{2}} \quad(z \in \mathbb{C} \backslash\{0\}) ; \\
E_{2,4}\left(z^{2}\right)=\frac{1}{z^{3}} \sinh z-\frac{1}{z^{2}} \quad(z \in \mathbb{C} \backslash\{0\}) ; \\
E_{2,6}\left(z^{2}\right)=\frac{1}{z^{5}} \sinh z-\frac{1}{z^{4}}-\frac{1}{6 z^{2}} \quad(z \in \mathbb{C} \backslash\{0\}) .
\end{gathered}
$$

Proof. From (50) and (52), we can obtain (64) and (65).

From (53) and (56), we can derive (66) and (67).

From (57) and (59), we can obtain (68) and (69).

From (60) and (63), we can find (70) and (71).

\section{Certain Relations among the Fox-Wright Functions}

In this section, by applying the Riemann-Liouville fractional integrals and derivatives to the recurrence relation (35), we obtain four relations among the Fox-Wright functions ${ }_{p} \Psi_{q}$, as stated in the following theorems. We also consider some particular cases of our main results.

Theorem 2. Let $\mu, \varrho, x \in \mathbb{R}^{+}$, and $a \in \mathbb{C}$. Then

$$
\begin{aligned}
& { }_{1} \Psi_{1}\left[\begin{array}{r}
(1,1) ; \\
(\mu+\varrho, \mu) ;
\end{array} x^{\mu}\right]-a x_{1}^{\mu} \Psi_{1}\left[\begin{array}{r}
(1,1) ; \\
(2 \mu+\varrho, \mu) ;
\end{array} x^{\mu}\right] \\
& =\varrho(\varrho+1)_{2} \Psi_{2}\left[\begin{array}{r}
(\varrho, \mu),(1,1) ; \\
(\varrho+2, \mu),(\mu+\varrho, \mu) ;
\end{array} x^{\mu}\right] \\
& -a \varrho(\varrho+1) x_{2}^{\mu} \Psi_{2}\left[\begin{array}{r}
(\mu+\varrho, \mu),(1,1) ; \\
(\mu+\varrho+2, \mu),(2 \mu+\varrho, \mu) ;
\end{array} x^{\mu}\right] .
\end{aligned}
$$

Proof. A particular case of (34) is

$$
\begin{gathered}
\int_{0}^{x} \tau^{\alpha-1}(x-\tau)^{\beta-1} \mathrm{~d} \tau=x^{\alpha+\beta-1} B(\alpha, \beta) \\
(x>0, \Re(\alpha)>0, \Re(\beta)>0) .
\end{gathered}
$$

Replacing $z$ by $a z^{\mu}$ in (35) and multiplying both sides of the resulting identity by $z^{\varrho-1}$, we obtain

$$
\begin{aligned}
z^{\varrho-1} E_{\mu, \varrho}\left(a z^{\mu}\right)= & \varrho(\varrho+1) z^{\varrho-1} E_{\mu, \varrho+2}\left(a z^{\mu}\right)-a \varrho(\varrho+1) z^{\mu+\varrho-1} E_{\mu, \mu+\varrho+2}\left(a z^{\mu}\right) \\
& +a z^{\mu+\varrho-1} E_{\mu, \mu+\varrho}\left(a z^{\mu}\right) .
\end{aligned}
$$


Taking the left-sided Riemann-Liouville fractional integral (12) on both sides of (74), we obtain

$$
\begin{aligned}
\left(I_{0+}^{\mu}\left[z^{\varrho-1} E_{\mu, \varrho}\left(a z^{\mu}\right)\right]\right)(x)= & \varrho(\varrho+1)\left(I_{0+}^{\mu}\left[z^{\varrho-1} E_{\mu, \varrho+2}\left(a z^{\mu}\right)\right]\right)(x) \\
& -a \varrho(\varrho+1)\left(I_{0+}^{\mu}\left[z^{\mu+\varrho-1} E_{\mu, \mu+\varrho+2}\left(a z^{\mu}\right)\right]\right)(x) \\
& +a\left(I_{0+}^{\mu}\left[z^{\mu+\varrho-1} E_{\mu, \mu+\varrho}\left(a z^{\mu}\right)\right]\right)(x) .
\end{aligned}
$$

Let $\mathcal{L}_{1}$ be the left-handed member of (75). Interchanging the integral and summation in (5), which may be verified under restrictions, we obtain

$$
\mathcal{L}_{1}=\frac{1}{\Gamma(\mu)} \sum_{k=0}^{\infty} \frac{a^{k}}{\Gamma(\mu k+\varrho)} \int_{0}^{x}(x-\tau)^{\mu-1} \tau^{\mu k+\varrho-1} \mathrm{~d} \tau
$$

Using (73) in the integral in (76), we obtain

$$
\mathcal{L}_{1}=x^{\mu+\varrho-1} \sum_{k=0}^{\infty} \frac{\Gamma(k+1)}{\Gamma(\mu k+\mu+\varrho)} \frac{\left(a x^{\mu}\right)^{k}}{k !}=x^{\mu+\varrho-1}{ }_{1} \Psi_{1}\left[\begin{array}{c}
(1,1) ; a x^{\mu} \\
(\mu+\varrho, \mu) ;
\end{array}\right]
$$

Now, let $\mathcal{R}_{1}$ be the right-handed member of (75). Similarly, as in obtaining (77), we derive

$$
\begin{gathered}
\mathcal{R}_{1}=\varrho(\varrho+1) x^{\mu+\varrho-1}{ }_{2} \Psi_{2}\left[\begin{array}{c}
(\varrho, \mu),(1,1) ; \\
(\varrho+2, \mu),(\mu+\varrho, \mu) ;
\end{array} a x^{\mu}\right] \\
-a \varrho(\varrho+1) x^{2 \mu+\varrho-1}{ }_{2} \Psi_{2}\left[\begin{array}{r}
(\mu+\varrho, \mu),(1,1) ; \\
(\mu+\varrho+2, \mu),(2 \mu+\varrho, \mu) ;
\end{array} a x^{\mu}\right] \\
+a x^{2 \mu+\varrho-1}{ }_{1} \Psi_{1}\left[\begin{array}{r}
(1,1) ; \\
(2 \mu+\varrho, \mu) ;
\end{array}\right] .
\end{gathered}
$$

Finally, equating the two identities in (77) and (78), we have (72).

Theorem 3. Let $x>0,0<\mu<1-\varrho$, and $a \in \mathbb{C}$. Then

$$
\begin{aligned}
& x_{2}^{\mu} \Psi_{2}\left[\begin{array}{r}
(1-\mu-\varrho, \mu),(1,1) ; \\
(\varrho, \mu),(1-\varrho, \mu) ;
\end{array} a x^{-\mu}\right] \\
& =\varrho(\varrho+1) x_{2}^{\mu} \Psi_{2}\left[\begin{array}{l}
\left.(1-\mu-\varrho, \mu),(1,1) ; a x^{-\mu}\right] \\
(\varrho+2, \mu),(1-\varrho, \mu) ;
\end{array}\right] \\
& -a \varrho(\varrho+1)_{2} \Psi_{2}\left[\begin{array}{r}
(1-\varrho, \mu),(1,1) ; \\
(\mu+\varrho+2, \mu),(\mu-\varrho+1, \mu) ;
\end{array}\right] \\
& +a_{2} \Psi_{2}\left[\begin{array}{r}
(1-\varrho, \mu),(1,1) ; \\
(\mu+\varrho, \mu),(\mu-\varrho+1, \mu) ;
\end{array} x^{-\mu}\right] .
\end{aligned}
$$

Proof. The following formula is readily derived:

$$
\begin{gathered}
\int_{x}^{\infty}(\tau-x)^{\alpha-1} \tau^{\beta-1} \mathrm{~d} \tau=x^{\alpha+\beta-1} B(\alpha, 1-\alpha-\beta) \\
\quad(x>0,0<\Re(\alpha)<1-\Re(\beta)) .
\end{gathered}
$$

Replacing $z$ by $a z^{-\mu}$ in (35) and multiplying both sides of the resulting identity by $z^{\varrho-1}$, we obtain

$$
\begin{aligned}
z^{\varrho-1} E_{\mu, \varrho}\left(a z^{-\mu}\right)= & \varrho(\varrho+1) z^{\varrho-1} E_{\mu, \varrho+2}\left(a z^{-\mu}\right)-a \varrho(\varrho+1) z^{-\mu+\varrho-1} E_{\mu, \mu+\varrho+2}\left(a z^{-\mu}\right) \\
& +a z^{-\mu+\varrho-1} E_{\mu, \mu+\varrho}\left(a z^{-\mu}\right) .
\end{aligned}
$$


Taking the right-sided Riemann-Liouville fractional integral (13) on both sides of (81), we obtain

$$
\begin{aligned}
\left(I_{\infty-}^{\mu}\left[z^{\varrho-1} E_{\mu, \varrho}\left(a z^{-\mu}\right)\right]\right)(x)= & \varrho(\varrho+1)\left(I_{\infty-}^{\mu}\left[z^{\varrho-1} E_{\mu, \varrho+2}\left(a z^{-\mu}\right)\right]\right)(x) \\
& -a \varrho(\varrho+1)\left(I_{\infty-}^{\mu}\left[z^{-\mu+\varrho-1} E_{\mu, \mu+\varrho+2}\left(a z^{-\mu}\right)\right]\right)(x) \\
& +a\left(I_{\infty-}^{\mu}\left[z^{-\mu+\varrho-1} E_{\mu, \mu+\varrho}\left(a z^{-\mu}\right)\right]\right)(x) .
\end{aligned}
$$

Now, similarly as in the proof of Theorem 2, applying (13) to each term of (82) and using (80), we can obtain the desired identity (79).

Theorem 4. Let $\mu, \varrho, x \in \mathbb{R}^{+}$. Further, let $a \in \mathbb{C}$ and $n=[\mu]+1 \in \mathbb{N}$. Then

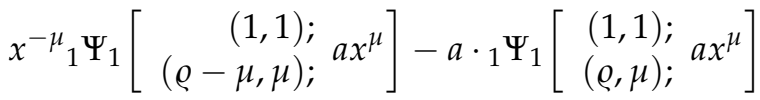

$$
\begin{aligned}
& =\varrho(\varrho+1) x_{2}^{-\mu_{2} \Psi_{2}}\left[\begin{array}{r}
(\varrho, \mu),(1,1) ; \\
(\varrho+2, \mu),(\varrho-\mu, \mu) ;
\end{array}\right] \\
& -a \varrho(\varrho+1)_{2} \Psi_{2}\left[\begin{array}{c}
(\mu+\varrho, \mu),(1,1) ; \\
(\mu+\varrho+2, \mu),(\varrho, \mu) ;
\end{array} a x^{\mu}\right] .
\end{aligned}
$$

Proof. Similar to (75), we have

$$
\begin{aligned}
\left(D_{0+}^{\mu}\left[z^{\varrho-1} E_{\mu, \varrho}\left(a z^{\mu}\right)\right]\right)(x)= & \varrho(\varrho+1)\left(D_{0+}^{\mu}\left[z^{\varrho-1} E_{\mu, \varrho+2}\left(a z^{\mu}\right)\right]\right)(x) \\
& -a \varrho(\varrho+1)\left(D_{0+}^{\mu}\left[z^{\mu+\varrho-1} E_{\mu, \mu+\varrho+2}\left(a z^{\mu}\right)\right]\right)(x) \\
& +a\left(D_{0+}^{\mu}\left[z^{\mu+\varrho-1} E_{\mu, \mu+\varrho}\left(a z^{\mu}\right)\right]\right)(x) .
\end{aligned}
$$

Let $\mathcal{L}$ be the left-handed member of (84). Using (14), (12), and (5), we obtain

$$
\begin{aligned}
\mathcal{L} & =\left(\frac{\mathrm{d}}{\mathrm{d} x}\right)^{n}\left\{\frac{1}{\Gamma(n-\mu)} \int_{0}^{x}(x-\tau)^{n-\mu-1} \tau^{\varrho-1} \sum_{k=0}^{\infty} \frac{a^{k}}{\Gamma(\mu k+\varrho)} \tau^{\mu k} \mathrm{~d} \tau\right\} \\
& =\left(\frac{\mathrm{d}}{\mathrm{d} x}\right)^{n}\left\{\frac{1}{\Gamma(n-\mu)} \sum_{k=0}^{\infty} \frac{a^{k}}{\Gamma(\mu k+\varrho)} \int_{0}^{x}(x-\tau)^{n-\mu-1} \tau^{\varrho+\mu k-1} \mathrm{~d} \tau\right\},
\end{aligned}
$$

under the restrictions of which integral and summation can be interchanged. Using (73) to evaluate the integral in (85) and interchanging differentiation and summation, we obtain

$$
\mathcal{L}=\sum_{k=0}^{\infty} \frac{a^{k}}{\Gamma(n-\mu+\varrho+\mu k)}\left(\frac{\mathrm{d}}{\mathrm{d} x}\right)^{n}\left(x^{n-\mu+\varrho+\mu k-1}\right) .
$$

Employing the following easily derivable formula

$$
\left(\frac{\mathrm{d}}{\mathrm{d} x}\right)^{n}\left(x^{\lambda}\right)=(-1)^{n}(-\lambda)_{n} x^{\lambda-n}=(-1)^{n} \frac{\Gamma(n-\lambda)}{\Gamma(-\lambda)} x^{\lambda-n}
$$

in (86), we find

$$
\mathcal{L}=x^{\varrho-\mu-1} \sum_{k=0}^{\infty} \frac{\left(a x^{\mu}\right)^{k}}{\Gamma(n-\mu+\varrho+\mu k)} \cdot(-1)^{n} \frac{\Gamma(1+\mu-\varrho-\mu k)}{\Gamma(1-n+\mu-\varrho-\mu k)} .
$$


Using the following well-known formula

$$
\Gamma(z) \Gamma(1-z)=\frac{\pi}{\sin (\pi z)} \quad(z \in \mathbb{C} \backslash \mathbb{Z})
$$

in (88), we derive

$$
\begin{aligned}
\mathcal{L} & =x^{\varrho-\mu-1} \sum_{k=0}^{\infty} \frac{\left(a x^{\mu}\right)^{k}}{\Gamma(\varrho-\mu+\mu k)} \\
& =x^{\varrho-\mu-1} \Psi_{1} \Psi_{1}\left[\begin{array}{c}
(1,1) ; \\
(\varrho-\mu, \mu) ;
\end{array} a x^{\mu}\right] .
\end{aligned}
$$

Further, the other three fractional derivatives in (84) can be evaluated as in (90). Finally, all of those evaluations that are used in (84) gives the desired identity (83).

Theorem 5. Let $\mu, \varrho, x \in \mathbb{R}^{+}$and $0<n-\mu<1-\varrho$. Further, let $a \in \mathbb{C}$ and $n=[\mu]+1 \in \mathbb{N}$. Then

$$
\begin{aligned}
& { }_{2} \Psi_{2}\left[\begin{array}{c}
(\mu-\varrho+1, \mu),(1,1) ; a x^{-\mu} \\
(\varrho, \mu),(1-\varrho, \mu) ;
\end{array}\right] \\
& =\varrho(\varrho+1)_{2} \Psi_{2}\left[\begin{array}{l}
\left.(1+\mu-\varrho, \mu),(1,1) ; a x^{-\mu}\right] \\
(\varrho+2, \mu),(1-\varrho, \mu) ;
\end{array}\right] \\
& -a \varrho(\varrho+1) x^{-\mu}{ }_{2} \Psi_{2}\left[\begin{array}{c}
(1+2 \mu-\varrho, \mu),(1,1) ; \\
(\mu+\varrho+2, \mu),(1+\mu-\varrho, \mu) ;
\end{array}\right] \\
& +a x_{2}^{-\mu} \Psi_{2}\left[\begin{array}{c}
\left.(1+2 \mu-\varrho, \mu),(1,1) ; a x^{-\mu}\right] . \\
(\mu+\varrho, \mu),(1+\mu-\varrho, \mu) ;
\end{array}\right] .
\end{aligned}
$$

Proof. Similar to (82), we have

$$
\begin{aligned}
\left(D_{\infty-}^{\mu}\right. & {\left.\left[z^{\varrho-1} E_{\mu, \varrho}\left(a z^{-\mu}\right)\right]\right)(x) } \\
= & \varrho(\varrho+1)\left(D_{\infty-}^{\mu}\left[z^{\varrho-1} E_{\mu, \varrho+2}\left(a z^{-\mu}\right)\right]\right)(x) \\
& -a \varrho(\varrho+1)\left(D_{\infty-}^{\mu}\left[z^{-\mu+\varrho-1} E_{\mu, \mu+\varrho+2}\left(a z^{-\mu}\right)\right]\right)(x) \\
& +a\left(D_{\infty-}^{\mu}\left[z^{-\mu+\varrho-1} E_{\mu, \mu+\varrho}\left(a z^{-\mu}\right)\right]\right)(x) .
\end{aligned}
$$

As in the proof of Theorem 4, by using (80), we can evaluate all of the four fractional derivatives in (92). For example,

$$
\left(D_{\infty-}^{\mu}\left[z^{\varrho-1} E_{\mu, \varrho}\left(a z^{-\mu}\right)\right]\right)(x)=x^{\varrho-\mu-1}{ }_{2} \Psi_{2}\left[\begin{array}{c}
(\mu-\varrho+1, \mu),(1,1) ; \\
(\varrho, \mu),(1-\varrho, \mu) ;
\end{array} a x^{-\mu}\right] .
$$

Hence, all the evaluations which are set in (92) yields the desired identity (91).

Since the results presented in Theorems $2-5$ are quite general, they may reduce to yield a number of interesting identities. For example, setting $\mu=1$ in the results, with the aid of (10), we obtain several new relations among the generalized hypergeometric functions $p F_{q}$, as stated in the following corollary (for current research of summation and reduction formulae for ${ }_{p} F_{q}$, see $\left.[68,69]\right)$.

Corollary 4. Let $a \in \mathbb{C}$. Then

$$
\begin{aligned}
& { }_{1} F_{1}\left[\begin{array}{r}
1 ; \\
\varrho+1 ;
\end{array} a x\right]-\frac{a x}{\varrho+1}{ }_{1} F_{1}\left[\begin{array}{r}
1 ; \\
\varrho+2 ;
\end{array} a x\right] \\
& ={ }_{2} F_{2}\left[\begin{array}{c}
\varrho, 1 ; \\
\varrho+2, \varrho+1 ;
\end{array} a x\right]-\frac{\varrho a x}{(\varrho+2)(\varrho+1)}{ }_{2} F_{2}\left[\begin{array}{c}
\varrho+1,1 ; \\
\varrho+3, \varrho+2 ;
\end{array} a x\right]
\end{aligned}
$$




$$
\begin{aligned}
& \left(x \in \mathbb{C}, \varrho \in \mathbb{C} \backslash \mathbb{Z}_{\leq-1}\right) ;
\end{aligned}
$$

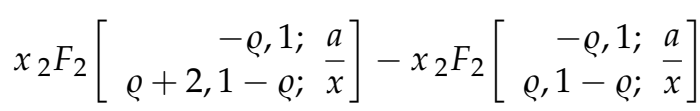

$$
\begin{aligned}
& =\frac{a \varrho}{(\varrho-1)(\varrho+2)}{ }_{2} F_{2}\left[\begin{array}{rr}
1-\varrho, 1 ; & a \\
\varrho+3,2-\varrho ; & x
\end{array}\right]-\frac{a}{\varrho-1}{ }_{2} F_{2}\left[\begin{array}{r}
1-\varrho, 1 ; \\
\varrho+1,2-\varrho ;
\end{array}\right] \\
& (x \in \mathbb{C} \backslash\{0\}, \varrho \in \mathbb{C} \backslash \mathbb{Z}) ; \\
& { }_{1} F_{1}\left[\begin{array}{r}
1 ; \\
\varrho-1 ;
\end{array} \text { ax }\right]-\frac{a x}{\varrho-1}{ }_{1} F_{1}\left[\begin{array}{l}
1 ; \\
\varrho ;
\end{array} a x\right] \\
& ={ }_{2} F_{2}\left[\begin{array}{r}
\varrho, 1 ; \\
\varrho+2, \varrho-1 ;
\end{array} a x\right]-\frac{\varrho a x}{(\varrho+2)(\varrho-1)}{ }_{2} F_{2}\left[\begin{array}{l}
\varrho+1,1 ; \\
\varrho+3, \varrho ;
\end{array} a x\right] ; \\
& \left(x \in \mathbb{C}, \varrho \in \mathbb{C} \backslash \mathbb{Z}_{\leq 1}\right) ; \\
& { }_{2} F_{2}\left[\begin{array}{rr}
2-\varrho, 1 ; & a \\
\varrho+2,1-\varrho ; & x
\end{array}\right]-{ }_{2} F_{2}\left[\begin{array}{rr}
2-\varrho, 1 ; & a \\
\varrho, 1-\varrho ; & x
\end{array}\right] \\
& =\frac{a(\varrho-2)}{x(\varrho-1)(\varrho+2)}{ }_{2} F_{2}\left[\begin{array}{r}
3-\varrho, 1 ; \\
\varrho+3,2-\varrho ;
\end{array}\right]-\frac{a(\varrho-2)}{x \varrho(\varrho-1)}{ }_{2} F_{2}\left[\begin{array}{rr}
3-\varrho, 1 ; & a \\
\varrho+1,2-\varrho ; & x
\end{array}\right] \\
& (x \in \mathbb{C} \backslash\{0\}, \varrho \in \mathbb{C} \backslash \mathbb{Z}) .
\end{aligned}
$$

It is worth noting that the restriction on each identity in Corollary 4 may be widened via analytic continuation. Further, each side of the identities (94) and (96) is easily checked to become 1 .

\section{Concluding Remarks and Posing Problems}

We reviewed the birth of the Mittag-Leffler function and its several extensions (among numerous ones) together with their diverse applications to a variety of research areas, particularly, fractional calculus. We recalled many known recurrence (or differential recurrence) relations for the two parametric Mittag-Leffler function (5) and the three parametric Mittag-Leffler function (7). Then, we established a number of recurrence relations for the two parametric Mittag-Leffler function (5). Further, by using appropriate pairings of those recurrence relations presented in this paper, we demonstrated that certain particular cases of the two parametric Mittag-Leffler function (5) can be expressed in terms of elementary functions. Further, by applying the Riemann-Liouville fractional integral and derivative operators to one of the recurrence relations for the two parametric Mittag-Leffler function (5), we derived four new relations among the Fox-Wright functions. Finally, we provided four relations among the generalized hypergeometric functions ${ }_{p} F_{q}$ as a particular case of those Fox-Wright function relations.

As in Section 3, by using the other formulas (36)-(41), we may derive some relations among the Fox-Wright functions ${ }_{p} \Psi_{q}$ together with their corresponding relations among 
the generalized hypergeometric functions ${ }_{p} F_{q}$ as particular cases. For example, as with Theorem 2, using (36), we obtain

$$
\begin{aligned}
& { }_{1} \Psi_{1}\left[\begin{array}{r}
(1,1) ; \\
(\mu+\varrho, \mu) ;
\end{array} x^{\mu}\right]-a^{3} x^{3 \mu}{ }_{1} \Psi_{1}\left[\begin{array}{r}
(1,1) ; \\
(4 \mu+\varrho, \mu) ;
\end{array} a x^{\mu}\right] \\
& =(\mu+\varrho) a x_{2}^{\mu} \Psi_{2}\left[\begin{array}{r}
\left.(\mu+\varrho, \mu),(1,1) ; a x^{\mu}\right] \\
(\mu+\varrho+1, \mu),(2 \mu+\varrho, \mu) ;
\end{array}\right] \\
& -(\mu+\varrho) a^{2} x^{2 \mu}{ }_{2} \Psi_{2}\left[\begin{array}{r}
(2 \mu+\varrho, \mu),(1,1) ; \\
(2 \mu+\varrho+1, \mu),(3 \mu+\varrho, \mu) ;
\end{array} x^{\mu}\right] \\
& +\frac{a^{2} x^{2 \mu}}{\Gamma(3 \mu+\varrho)}+\frac{1}{\Gamma(\mu+\varrho)} \\
& \left(\mu, \varrho, x \in \mathbb{R}^{+}, a \in \mathbb{C}\right)
\end{aligned}
$$

and

$$
\begin{aligned}
{ }_{1} F_{1}\left[\begin{array}{c}
1 ; \\
1+\varrho ;
\end{array} a x\right]-\frac{a^{3} x^{3}}{(\varrho+1)(\varrho+2)(\varrho+3)}{ }_{1} F_{1}\left[\begin{array}{r}
1 ; \\
\varrho+4 ;
\end{array} a x\right] \\
=\frac{a x}{\varrho+1}{ }_{2} F_{2}\left[\begin{array}{l}
\varrho+1,1 ; \\
\varrho+2, \varrho+2 ;
\end{array} a x\right]-\frac{a^{2} x^{2}}{(\varrho+2)^{2}}{ }_{2} F_{2}\left[\begin{array}{r}
\varrho+2,1 ; \\
\varrho+3, \varrho+3 ;
\end{array} a x\right] \\
\quad+\frac{a^{2} x^{2}}{(\varrho+1)(\varrho+2)}+1 \quad\left(\varrho \in \mathbb{C} \backslash \mathbb{Z}_{\leq-1}, a, x \in \mathbb{C}\right) .
\end{aligned}
$$

Here we pose the following problems:

(i) Based on the other recurrence relations (36)-(41), by using Riemann-Liouville fractional integral and derivative operators, try to present certain relations among the Fox-Wright functions ${ }_{p} \Psi_{q}$;

(ii) Demonstrate some particular cases of the identities given in (i);

(iii) Based on the recurrence relations (35)-(41), by using the other fractional integral and derivative operators, try to present certain relations among some special functions;

(iv) Consider certain particular cases of identities that will be derived in (iii).

Author Contributions: Writing—original draft, D.S.S., S.J. and J.C.; Writing—review and editing, D.S.S., S.J. and J.C. All authors have read and agreed to the published version of the manuscript.

Funding: The third-named author was supported by the Basic Science Research Program through the National Research Foundation of Korea (NRF) funded by the Ministry of Education (NRF2020R111A1A01052440).

Institutional Review Board Statement: Not applicable.

Informed Consent Statement: Not applicable.

Data Availability Statement: Not applicable.

Acknowledgments: The authors are very thankful of the anonymous referees' constructive and encouraging comments, which helped to improve this article.

Conflicts of Interest: The authors have no conflict of interest.

\section{References}

1. Mittag-Leffler, M.G. Sur l'intégrale de Laplace-Abel. C. R. Acad. Sci. Paris (Ser. II) 1902, 136, 937-939.

2. Mittag-Leffler, M.G. Sur la nouvelle fonction $E_{\alpha}(x)$. C. R. Acad. Sci. Paris (Ser. II) 1903, 137, 554-558.

3. Mittag-Leffler, M.G. Sopra la funzione $E_{\alpha}(x)$. Rend. Accad. Lincei 1904, 13, 3-5.

4. Buhl, A. Séries Analytiques, Sommabilité, Number 7 in Mémorial des Sciences Mathématiques; Gauthier-Villars: Paris, France, 1925.

5. Srivastava, H.M.; Choi, J. Zeta and q-Zeta Functions and Associated Series and Integrals; Elsevier Science Publishers: Amsterdam, The Netherlands; London, UK; New York, NY, USA, 2012.

6. Gorenflo, R.; Kilbas, A.A.; Mainardi, F.; Rogosin, S. Mittag-Leffler Functions, Related Topics and Applications, 2nd ed.; Springer: Berlin, Germany, 2020. 
7. Abramowitz, M.; Stegun, I.A. (Eds.) Handbook of Mathematical Functions with Formulas, Graphs, and Mathematical Tables; Applied Mathematics Series 55, Ninth Printing; National Bureau of Standards: Washington, DC, USA, 1972; Reprint of the 1972 Edition, Dover Publications, Inc.: New York, NY, USA, 1992.

8. Djrbashian, M.M. Harmonic Analysis and Boundary Value Problems in the Complex Domain; Birkhauser Verlay: Basel, Switzerland; Boston, MA, USA; Berlin, Germany, 1996.

9. Djrbashian, M.M. Integral Transforms and Representations of Functions in the Complex Domain; Nauka: Moscow, Russia, 1966.

10. Erdélyi, A.; Magnus, W.; Oberhettinger, F.; Tricomi, F.G. Higher Transcendental Functions; McGraw-Hill Book Company: New York, NY, USA; Toronto, ON, Canada; London, UK, 1955; Volume 3.

11. Gorenflo, R.; Luchko, Y.; Rogosin, S. Mittag-Leffler Type Functions: Notes on Growth Properties and Distribution of Zeros; Preprint No. A04-97; Serie A Mathematik; Freie Universität Berlin: Berlin, Germany, 1997.

12. Gorenflo, R.; Mainardi, F. Fractional Oscillations and Mittag-Leffler Type Functions; Preprint No. A14-96; Freie Universität Berlin, Serie A Mathematik; Berlin, Germany, 1996.

13. Gorenflo, R.; Mainardi, F. The Mittag-Leffler type functions in the Riemann-Liouville fractional calculus. In Boundary Value Problems, Special Functions and Fractional Calculus; Kilbas, A.A., Ed.; Proceedings of International Conference (Minsk, 1996); Belarusian State University: Minsk, Belarus, 1996; pp. 215-225.

14. Mittag-Leffler, G. Sur la représentation analytique d'une branche uniforme d'une fonction monogène (cinquième note). Acta Math. 1905, 29, 101-181. [CrossRef]

15. Wiman, A. Über den fundamentalsatz in der theorie der funktionen $E_{\alpha}(x)$. Acta Math. 1905, 29, 191-201. [CrossRef]

16. Wiman, A. Über die nullsteliun der funktionen $E_{\alpha}(x)$. Acta Math. 1905, 29, 217-234. [CrossRef]

17. Mainardi, F. Why the Mittag-Leffler Function can be considered the queen function of the fractional calculus? Entropy 2020, 22, 1359. [CrossRef]

18. Mathai, A.M.; Saxena, R.K. The H-Function with Applications in Statistics and Other Disciplines; Halsted Press (John Wiley \& Sons): New York, NY, USA; London, UK; Sydney, Australia; Toronto, ON, Canada, 1978.

19. Mathai, A.M.; Saxena, R.K.; Haubold, H.J. The H-Function: Theory and Applications; Springer: New York, NY, USA, 2010.

20. Bagley, R.L. On the fractional order initial value problem and its engineering applications. In Fractional Calculus and Its Applications; Proceedings of International Conference (Tokyo, 1989); College of Engineering, Nihon University: Tokyo, Japan, 1990; pp. 12-20.

21. Beyer, Y.; Kempfle, S. Definition of physically consistent damping laws with fractional derivatives. Z. Angew. Math. Mech. 1995, 75, 623-635. [CrossRef]

22. Caputo, M.; Mainardi, F. Linear models of dissipation in anelastic solids. Riv. Nuovo Cimento 1971, 1, 161-198. [CrossRef]

23. Goldsmith, P.L. The calculation of true practicle size distributions from the sizes observed in a thin slice. Br. J. Appl. Phys. 1967, 18, 813-830. [CrossRef]

24. Haubold, H.J.; Mathai, A.M.; Saxena, R.K. Mittag-Leffler functions and their applications. J. Appl. Math. 2011, $2011,298628$. [CrossRef]

25. Nonnenmacher, T.F.; Glockle, W.G. A fractional model for mechanical stress relaxation. Philos. Mag. Lett. 1991, 64, 89-93. [CrossRef]

26. Schneider, W.R.; Wyss, W. Fractional diffusion and wave equations. J. Math. Phys. 1989, 30, 134-144. [CrossRef]

27. Humbert, P.; Agrawal, R.P. Sur la fonction de Mittag-Leffler et quelques-unes de ses généralisations. Bull. Sci. Math. 1953, 77, 180-185.

28. Hille, E.; Tamarkin, J.D. On the theory of linear integral equations. Ann. Math. 1930, 31, 479-528. doi: 10.2307/1968241. [CrossRef]

29. Kilbas, A.A.; Saigo, M. On solution of integral equations of Abel-Volterra type. Differ. Integral Equ. 1995, 8, 993-1011.

30. Kilbas, A.A.; Saigo, M. On Mittag-Leffler type function, fractional calculus operators and solutions of integral equations. Integral Transform. Spec. Funct. 1996, 4, 355-370. [CrossRef]

31. Kilbas, A.A.; Saigo, M. Fractional integrals and derivatives of Mittag-Leffler type function (Russian). Doklady Akad. Nauk Belarusi 1995, 39, 22-26.

32. Kilbas, A.A.; Saigo, M. Solution of Abel type integral equations of second kind and differential equations of fractional order (Russian). Doklady Akad. Nauk Belarusi 1995, 39, 29-34.

33. Miller, K.S.; Ross, B. An Introduction to the Fractional Calculus and Fractional Differential Equations; Wiley: New York, NY, USA, 1993.

34. Samko, S.G.; Kilbas, A.A.; Marichev, O.I. Fractional Integrals and Derivatives: Theory and Applications; Gordon and Breach Science Publishers: Yverdon, Switzerland; Amsterdam, The Netherlands; Reading, UK; Tokyo, Japan; Paris, France; Berlin, Germany; Langhorne, PA, USA; Victoria, Australia, 1993.

35. Choi, J.; Parmar, R.K.; Chopra, P. Extended Mittag-Leffler function and associated fractional calculus operators. Georgian Math. J. 2019, 27, 11. [CrossRef]

36. Mainardi, F.; Gorenflo, R. Time-fractional derivatives in relaxation processes: A tutorial survey. Fract. Calc. Appl. Anal. 2007, 10, 269-308.

37. Agarwal, R.P. A propos d'une note de M. Pierre Humbert. CR Acad. Sci. Paris 1953, 236, 2031-2032.

38. Fry, C.G.; Hughes, H.K. Asymptotic developments of certain integral functions. Duke Math. J. 1942, 9, 791-802. [CrossRef]

39. Humbert, P. Quelques resultatsrelatifs a la fonction de Mittag-Leffler. CR Acad. Sci. Paris 1953, 236, 1467-1468.

40. Schneider, W.R. Completely monotone generalized Mittag-Leffler functions. Expo. Math. 1996, 14, 3-24.

41. Sedletskii, A.M. Asymptotic formulas for zero of a function of Mittag-Leffler type (Russian). Anal. Math. 1994, 20, 117-132. 
42. Giusti, A.; Colombaro, I.; Garra, R.; Garrappa, R.; Polito, F.; Popolizio, M.; Mainardi, F. A practical guide to Prabhakar fractional calculus. Fract. Calc. Appl. Anal. 2020, 23, 9-54. [CrossRef]

43. Prabhakar, T.R. A singular integral equation with a generalized Mittag-Leffler function in the kernel. Yokohama Math. J. 1971, 19, 7-15.

44. Wright, E.M. The asymptotic expansion of the generalized hypergeometric function. Proc. Lond. Math. Soc. 1940, 46, 389-408. [CrossRef]

45. Wright, E.M. The asymptotic expansion of integral functions defined by Taylor series. Philos. Trans. R. Soc. Lond. A 1940, 238, 423-451. [CrossRef]

46. Wright, E.M. The asymptotic expansion of integral functions defined by Taylor series (second paper). Philos. Trans. R. Soc. Lond. A 1941, 239, 217-232. [CrossRef]

47. Fox, C. The asymptotic expansion of generalized hypergeometric functions. Proc. Lond. Math. Soc. 1928, 27, 389-400. [CrossRef]

48. Srivastava, H.M.; Karlsson, P.W. Multiple Gaussian Hypergeometric Series; Halsted Press (Ellis Horwood Limited): Chichester, UK; John Wiley and Sons: New York, NY, USA; Chichester, UK; Brisbane, Australia; Toronto, ON, Canada, 1985.

49. Kilbas, A.A.; Srivastava, H.M.; Trujillo, J.J. Theory and Applications of Fractional Differential Equations; North-Holland Mathematical Studies, Elsevier (North-Holland) Science Publishers: Amsterdam, The Netherlands; London, UK; New York, NY, USA, 2006; Volume 204.

50. Srivastava, H.M.; Tomovski, Ž. Fractional calculus with an integral operator containing a generalized Mittag-Leffler function in the kernel. Appl. Math. Comput. 2009, 211, 198-210. [CrossRef]

51. Gupta, I.S.; Debnath, L. Some properties of the Mittag-Leffler functions. Integral Transform. Spec. Funct. 2007, 18, 329-336. [CrossRef]

52. Saxena, R.K. Certain properties of generalized Mittag-Leffler function. In Proceedings of the 3rd Annual Conference of the Society for Special Functions and Their Applications, Chennai, India, 22 June-3 July 2002; pp. 77-81.

53. Hilfer, R.; Seybold, H.J. Computation of the generalized Mittag-Leffler function and its inverse in the complex plane. Integral Transform. Spec. Funct. 2006, 17, 637-652. [CrossRef]

54. Podlubny, I. Fractional Differential Equations; Academic Press: San Diego, CA, USA; Boston, MA, USA; New York, NY, USA; London, UK; Sydney, Australia; Tokyo, Japan; Toronto, ON, Canada, 1999.

55. Seybold, H.; Hilfer, R. Numerical Algorithm for calculating the generalized Mittag-Leffler function. SIAM J. Numer. Anal. 2008, 47, 69-88. [CrossRef]

56. Berberan-Santos, M.N. Properties of the Mittag-Leffler relaxation function. J. Math. Chem. 2005, 38, 629-635. [CrossRef]

57. Garra, R.; Garrappa, R. The Prabhakar or three parameter Mittag-Leffler function: Theory and application. Commun. Nonlinear Sci. Numer. Simul. 2018, 56, 314-329. [CrossRef]

58. Shukla, A.K.; Prajapati, J.C. On a recurrence relation of generalized Mittag-Leffler function. Surv. Math. Appl. 2009, 4 133-138.

59. Shukla, A.K.; Prajapati, J.C. On a generalization of Mittag-Leffler function and its properties. J. Math. Anal. Appl. 2007, 336, 797-811. [CrossRef]

60. Salim, T.O. Some properties relating to the generalized Mittag-Leffler function. Adv. Appl. Math. Anal. 2009, 4, 21-30.

61. Kurulay, M.; Bayram, M. Some properties of the Mittag-Leffler functions and their relation with the Wright functions. Adv. Diff. Equ. 2012, 2012, 181. [CrossRef]

62. Dhakar, V.S.; Sharma, K. On a recurrence relation of K-Mittag-Leffler function. Commun. Korean Math. Soc. 2013, 28, 851-856. [CrossRef]

63. Dorrego, G.A.; Cerutti, R.A. The k-Mittag-Leffler function. Int. J. Contemp. Math. Sci. 2012, 7, 705-716.

64. Díaz, R.; Pariguan, E. On hypergeometric functions and k-Pochhammer symbol. Divulg. Mat. 2007, 15, 179-192.

65. Sharma, S.; Jain, R. On some recurrence relations of generalized q-Mittag Leffler function. Math. Aeterna 2016, 6, 791-795.

66. Gehlot, K.S. Recurrence relation and integral representation of $p-k$ Mittag-Leffler function. Palest. J. Math. 2021, 10, $290-298$.

67. Gehlot, K.S. The $p-k$ Mittag-Leffler function. Palest. J. Math. 2018, 7, 628-632.

68. Choi, J. Certain applications of generalized Kummer's summation formulas for ${ }_{2} F_{1}$. Symmetry 2021, 13, 1538. [CrossRef]

69. Choi, J.; Qureshi, M.I.; Bhat, A.H.; Majid, J. Reduction formulas for generalized hypergeometric series associated with new sequences and applications. Fractal Fract. 2021, 5, 150. [CrossRef] 